\title{
Two Types of X-ray Spectra in Cataclysmic Variables
}

\author{
K. Mukai
}

NASA/GSFC and USRA, Code 662, NASA/Goddard Space Flight Center, Greenbelt, MD 20771, USA.

\author{
A. Kinkhabwala, J.R. Peterson, S.M. Kahn, F. Paerels \\ Columbia Astrophysics Laboratory, Columbia University, 550 West \\ 120th Street, New York, New York 10027, USA.
}

\begin{abstract}
We present Chandra HETG spectra of seven cataclysmic variables, including four intermediate polars. We find that they divide unambiguously into two distinct types. Spectra of the first type (nonmagnetic systems and EX Hya) are remarkably well fit by a simple cooling flow model, which assumes only steady-state isobaric radiative cooling. The maximum temperature, $k T_{\max }$, and the normalization, which provides a highly precise measurement of the accretion rate, are the only free parameters of this model. Spectra of the second type (the three other IPs) are grossly inconsistent with a cooling flow model. They instead exhibit a hard continuum, and show strong H-like and He-like ion emission but little Fe L-shell emission, which is consistent with expectations for line emission from a photoionized plasma. Using a simple photoionization model, we argue that the observed line emission for these sources can be driven entirely by the hard continuum. The physical significance of these two distinct types of X-ray spectra is also explored.
\end{abstract}

\section{Introduction}

$\mathrm{X}$-ray observations of cataclysmic variables (CVs) probe the physics of accretion very near the white dwarf. In magnetic CVs (MCVs), accretion is expected to be quasi-radial, with a strong standing shock. The shock heats the plasma to high temperatures $\left(k T_{\max } \sim 10-50 \mathrm{keV}\right)$, and must cool before it can settle onto the white dwarf surface (see, e.g., Aizu 1973). This cooling process is best observed in the $\sim 0.5-10 \mathrm{keV}$ band, where a host of emission lines are expected from the cooling plasma. Such lines were indeed detected in EX Hya using the CCD-based Solid-state Imaging Spectrometer (SIS) instrument on-board the Japanese ASCA mission (Ishida, Mukai \& Osborne 1994). However, later observations of other MCVs were rather disappointing in this respect; in a study of 23 MCVs, Ezuka \& Ishida (1999) find that only 3 systems (EX Hya, AE Aqr, 
and V884 Her) require multi-temperature components ${ }^{1}$. For some systems, poor data quality and the partial covering absorber can be blamed for this; however, for others (e.g., AM Her: Ishida et al. 1997), this excuse cannot be used, and the lack of signatures of lower temperature plasma has become a significant puzzle.

With the availability of Chandra high-energy transmission gratings (HETG), sensitive to X-rays in the $1.5-25 \AA(\sim 0.5-8 \mathrm{keV})$ range with unprecedented spectral resolution, we have the opportunity to re-examine this question. We have just concluded our initial study of magnetic and non-magnetic CVs (Mukai et al. 2003). Here we reproduce our basic findings and discuss implications for the study of MCVs.

\section{Observations and Spectra}

Seven CVs were observed with Chandra HETG by 2002 March; we have obtained these data from the archive and analyzed them using CIAO 2.2.1. The objects are four intermediate polars (IPs) EX $\mathrm{Hya}^{2}$, V1223 Sgr, AO Psc, and GK Per, two dwarf novae SS Cyg and U Gem, and one old nova V603 Aql. For CVs with multiple HETG observations, we have chosen to analyze one spectrum per system (quiescent spectrum of SS Cyg, and one of two similar spectra of GK Per, both during the 2002 outburst).

These seven X-ray spectra divide unambiguously into two types. Spectra of the first type are well fit by a simple cooling flow model, whereas spectra of the second type are well described by a simple model of a photoionized plasma. Combined first-order $(m= \pm 1)$ MEG spectra of cooling flow CVs are shown in Fig. 1 and discussed in $\S 3.1$; the spectra of photoionized CVs are shown in Fig. 2 and discussed in $§ 3.2$.

\section{Spectral Analysis}

\subsection{Cooling Flow Spectra}

The four CV spectra of EX Hya, V603 Aql, U Gem, and SS Cyg shown in Fig. 1 are strikingly similar. These spectra exhibit a smooth continuum, strong $\mathrm{H}$ - and He-like ion emission lines of $\mathrm{O}, \mathrm{Ne}, \mathrm{Mg}, \mathrm{Al}, \mathrm{Si}$, and $\mathrm{S}$, and strong emission from ions spanning the entire Fe L-shell complex (FeXVII - Fe XXIV). In the HEG spectra (not shown), H- and He-like Fe lines are strong, while the intensity of the Fe fluorescence line varies from system to system.

The observed wide range and strengths of emission from $\mathrm{H}$ - and He-like ions, the level and shape of the continuum (suggesting bremsstrahlung), and, in particular, the comparable strengths of emission across the full range of $\mathrm{Fe} \mathrm{L}$ shell ions are all consistent with expectations for a multitemperature thermal plasma with a relatively flat emission measure distribution. The flatness of this distribution is further indicative of an isobaric cooling flow, which assumes only

\footnotetext{
${ }^{1}$ In addition, YY Dra, which was not included in their study, also requires multi-temperature components.
}

${ }^{2}$ See also Mauche, Liedahl \& Fournier (2001) and Mauche (2002) 


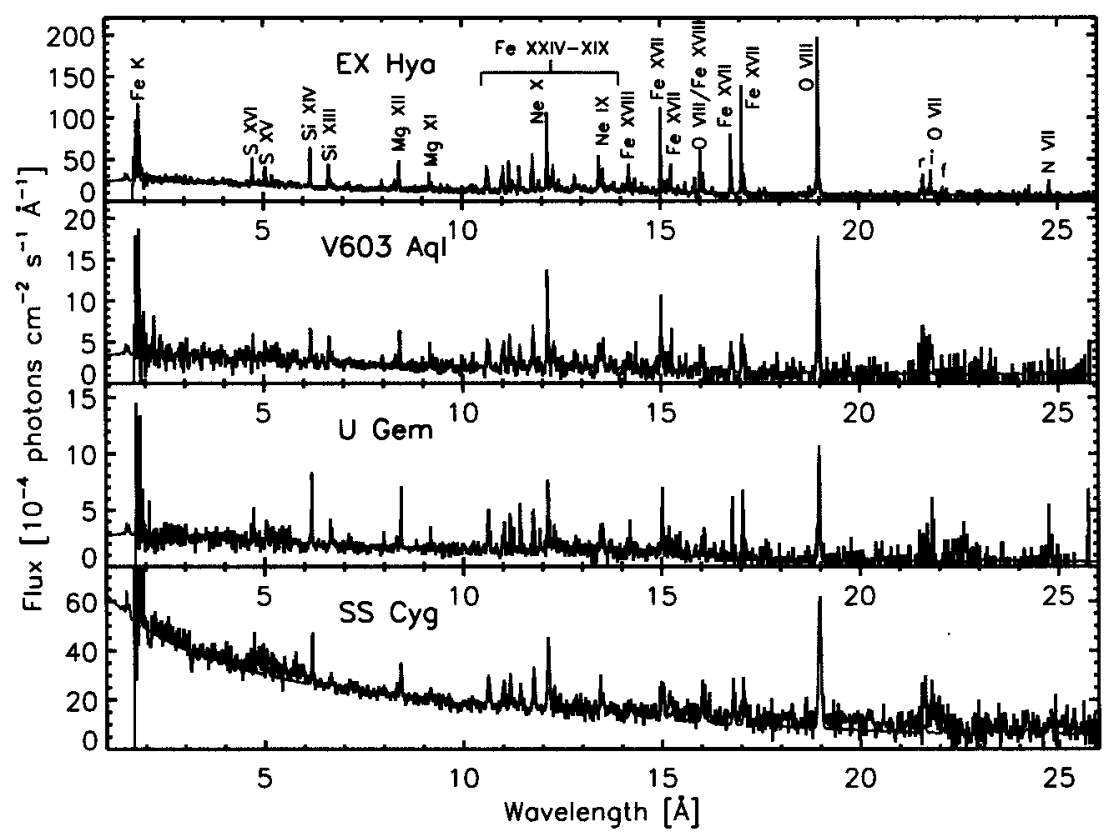

Figure 1. Chandra HETG spectra (MEG, $m= \pm 1$ orders) of 4 CVs that exhibit cooling flow spectra. Both data and models are shown. See Mukai et al. (2003) for a color version of this figure.

that the gas releases all of its energy in the form of optically-thin radiation as it cools in a steady-state flow. The two main parameters of this model are the maximum temperature, $k T_{\max }$, and the overall normalization, which directly gives the total mass flow rate. Fits to the spectra using mkcflow (with a uniform velocity broadening and an interstellar absorber) in xspec are shown in Fig. 1 (the model and data overlap and are virtually indistinguishable in this black and white version; see Mukai et al. 2003 for a color version of this figure). We obtain the same $k T_{\max }$ of $20 \mathrm{keV}$ for EX Hya, V603 Aql, and U Gem, but a $k T_{\max }$ of $80 \mathrm{keV}$ for SS Cyg, accounting for the stronger continuum in this source. We caution, however, that the fits of Chandra HETG data alone using mkcflow may not give a reliable value of true $k T_{\max }$ (for example, overall metal abundances can also affect the continuum to line ratio). These high resolution observations unambiguously demonstrate that post-shock plasmas in some CVs are cooling flows, as originally pointed out by Fabian \& Nulsen (1977) and applied to CVs by Done, Osborne \& Beardmore (1995).

\subsection{Photoionized Spectra}

The spectra shown in Fig.2 are noticeably different from those shown in Fig.1, but are very similar to one another. In particular, V1223 Sgr and AO Psc both have a hard, power-law-like continuum, and strong lines from medium $\mathrm{Z}$ elements, but the Fe L-shell emission is limited only to FeXVII lines, with no 


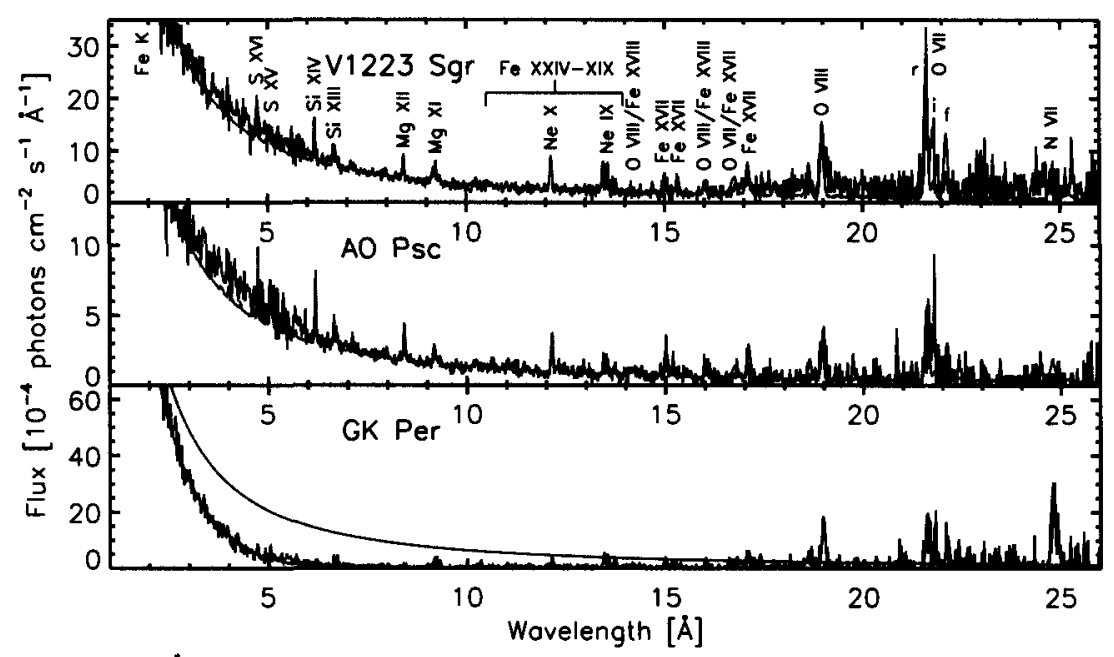

Figure 2. Chandra HETG spectra (MEG, $m= \pm 1$ orders) of 3 IPs that exhibit photoionized spectra. Data are shown as histograms, models as lines; an additional line for GK Per is the inferred intrinsic continuum. See Mukai et al. (2003) for a color version.

detectable Fe XVIII-Fe XXIV lines. This does not indicate a low Fe abundance, since we detect strong $\mathrm{K} \alpha$ lines (H-like, He-like, and fluorescence) in the HEG spectra (not shown). GK Per has a continuum that is harder still, and shows $\mathrm{H}$ - and He-like ions but little Fe L-shell emission. This type of emission line spectrum is incompatible with cooling flow plasma. Instead, it resembles the expectations for photoionized plasma (Sako et al. 2000), and the observed spectrum of the prototype Seyfert 2, NGC 1068, for which photoionization origin has been demonstrated Kinkhabwala et al. (2002).

We model these spectra with the xspec photoionization model photoion ${ }^{3}$ (Kinkhabwala et al. 2002). We assume that the observed continuum of V1223 Sgr and AO Psc is that which drives the line emission, while a similar continuum (shown as an additional line in Fig.2) drives the photoionization in GK Per but is obscured along our particular line of sight. We also assume the line emission is unabsorbed in all three systems. Furthermore, we assume that all lines remain completely unsaturated at all ionic column densities, due to radial velocity distribution widths of thousands of $\mathrm{km} \mathrm{s}^{-1}$. This last assumption is necessary to explain the strong resonance lines in the He-like triplets, which are not predicted by traditional photoionization models (Porque \& Dubau 2000). With these assumptions, we find that a simple model of a photoionized cone of plasma fits the data very well. Estimated lower limits to the accretion rates necessary to power the photoionizing continuum are similar to the mass flow rates in the cooling-flow CVs.

\footnotetext{
${ }^{3}$ http://xmm.astro.columbia.edu/photoion/photoion.html
} 


\section{Discussion}

We have shown that a simple cooling flow model is capable of reproducing the X-ray spectra of four CVs including EX Hya, while a photoionization model can successfully reproduce the strikingly different spectra of three other IPs. This finding leads to a series of questions. (1) Is cooling flow the correct model for the four systems? (2) Why is EX Hya similar to non-magnetic CVs? (3) Why is EX Hya dissimilar from other IPs? And (4) Where is the cooling flow in GK Per, AO Psc, and V1223 Sgr?

Cooling Flow model: We have used a model developed for clusters of galaxies to fit the CV spectra. This model ignores additional cooling mechanisms (see, e.g., Wu, Chanmugam \& Shaviv 1994), and the reflection off the white dwarf surface (Beardmore et al. 1995). These effects, however, are important at high energies that Chandra HETG is less sensitive, hence do not severely affect our study. On the other hand, mkcflow includes a proper treatment of line cooling, which is the dominant cooling mechanism for $\mathrm{kT} \sim 1 \mathrm{keV}$ plasma (Gehrels \& Williams 1993) but is often ignored in analytical treatments. At the lowest temperature regions, the complexities due to the lower boundary conditions (Wu \& Cropper 2001) should be taken into consideration; the fact that our fits are successful suggests that this effect is not important for plasma temperatures above $\sim 2$ million degrees in these 4 systems (the O VII triplet has peak emissivity near $2 \times 10^{6} \mathrm{~K}$, the lowest among all securely detected lines).

Similarity of EX Hya to non-magnetic CVs: Once we adopt the cooling flow prescriptions, with the caveats given above, there is only a limited number of free parameters. Of these, the shock temperature $\left(k T_{\max }\right)$ can be altered by the accretion geometry (column vs. boundary layer). However, it appears that any such effect does not amount to more than a factor of several, and that $k T_{\max }$ is largely determined by the depth of the gravitational potential.

Uniqueness of EX Hya?: The difference between the two X-ray spectral types may be the specific accretion rate (accretion rate per unit area). In most IPs, the magnetic field collimates the flow onto small regions $(<0.2 \%$ of the white dwarf surface in XY Ari: Hellier 1997). EX Hya, however, is thought to have a much lower specific accretion rate than typical IPs, creating a tall, lower density, shock (Allan, Hellier \& Beardmore 1998). The resultant top-hat geometry of the postshock region will allow the cooling flow X-rays to escape the post-shock region from the side without having to travel through the pre-shock flow ${ }^{4}$. Based on the ASCA spectra (Ezuka \& Ishida 1999) and other observational clues, we speculate that, among MCVs, AE Aqr, YY Dra, and V884 Her may also have cooling flow spectra. On the other hand, typical IPs such as V1223 Sgr and AO Psc probably have a high specific accretion rate, leading to a pill-box geometry which does not allow the cooling flow spectrum to escape freely.

Cooling Flows in IPs: We consider it likely that these three systems are also powered by the same cooling flow-type emission from the post shock region. Indeed, the 2-20 keV spectra of V1223 Sgr and AO Psc have been successfully

\footnotetext{
${ }^{4}$ The specific accretion rate in all non-magnetic CVs may be low because the boundary layer accretion probably occurs over a much larger fraction of the white dwarf surface than the polar spots in MCVs.
} 
fit with Aizu-type models (Cropper, Ramsay \& Wu 1998; Ramsay 2000). There are several effects that can potentially operate to hide the cooling flow emission signatures in the Chandra data of these systems. One is the lower boundary condition mentioned above. Second, the post-shock region may have a high optical depth to lines. The low-temperature cut-off used by Beardmore, Osborne \& Hellier (2000) for ASCA SIS spectrum of V1223 Sgr (of the order of $2 \mathrm{keV}$ ) may reflect one of the two effects. Third, in a pill-box geometry, our line of sight must pass through the immediate pre-shock flow, which can absorb much of the soft emissions (Done \& Magdziarz 1998). This is suspected to be the main site of intrinsic absorption in IPs, rather than the more distant part of the accretion curtain (Hellier et al. 1996).

We thus explain the Chandra HETG spectra of three IPs as differentially absorbed cooling flow continuum, plus photoionized emission lines from the immediate pre-shock flow. A power-law distribution of absorption column Done \& Magdziarz (1998) can produce the hard power-law continuum observed in these systems. As for the lines, the possibility of photoionized emission lines around $1 \mathrm{keV}$ was first pointed out by Kallman et al. (1993).

\section{Future Prospects}

The vastly improved quality of the Chandra HETG spectra leaves little doubt that the spectrum of EX Hya is very different from those of V1223 Sgr, AO Psc, and GK Per. However, our interpretation of the latter is admittedly somewhat tentative and further modelling is necessary. In particular, it is a puzzle how the continuum of V1223 Sgr and AO Psc are so similar, and why that of GK Per is different. Along with theoretical works, observations of other MCVs with grating spectrometers on Chandra and XMM-Newton, and observations of one or more systems of sufficient quality to allow phase-resolved spectroscopy are clearly desirable. The high resolution, non-dispersive X-Ray Spectrometer (XRS) instrument on-board the Japanese Astro-E2 mission has much promise for the latter.

\section{References}

Aizu, K. 1973, Prog. Theor. Phys., 49, 1184

Allan, A., Hellier, C., \& Beardmore, A.P. 1998, MNRAS, 295, 167

Beardmore, A.P., Done, C., Osborne, J.P., \& Ishida, M. 1995, MNRAS, 272, 749

Beardmore, A.P., Osborne, J.P., \& Hellier, C. 2000, MNRAS315, 307

Cropper, M., Ramsay, G. \& Wu, K. 1998, MNRAS, 293, 222

Done, C., Osborne, J.P., \& Beardmore, A.P. 1995, MNRAS, 276, 483

Done, C., \& Magdziarz, P. 1998, MNRAS, 298, 737

Ezuka, H., \& Ishida, M. 1999, ApJS, 120, 277

Fabian, A.C., Nulsen, P.E.J. 1977, MNRAS, 180, 479

Gehrels, N., \& Williams, E.D. 1993, ApJ, 418, L25

Hellier, C 1997, MNRAS, 291, 71 
Hellier, C., Mukai, K., Fujimoto, R., \& Ishida, M. 1996, MNRAS, 280, 877

Ishida, M., Mukai, K., \& Osborne, J.P. 1994, PASJ, 46, L81

Ishida, M., Matsuzaki, K., Fujimoto, R., Mukai, K., \& Osborne, J.P. 1997, MNRAS, 287, 651

Kallman, T.R., Schlegel, E.M., Serlemitsos, P.J. (+13 co-authors) 1993, ApJ, 411,869

Kinkhabwala, A., Sako, M., Behar, E., Kahn, S.M., Paerels, F.B.S., Brinkman, A.C., Kaastra, J.S., Gu, M.F., \& Liedahl, D.A. 2002, ApJ, 575, 732

Mauche, C.W. 2002, in "The Physics of Cataclysmic Variables and Related Objects," eds. B.T. Gänsicke, K. Beuermann, \& K. Reinsch (San Francisco: Astronomical Society of the Pacific), 113

Mauche, C.W., Liedahl, D.A., \& Fournier, K.B. 2001, ApJ, 560, 992

Mukai, K., Kinkhabwala, A., Peterson, J.R., Kahn, S.M., \& Paerels, F. 2003, ApJ, 586, L77

Porquet, D., \& Dubau, J. 2000, A\&AS, 143, 495

Ramsay, G. 2000, MNRAS, 314, 403

Sako, M., Kahn, S.M., Paerels, F.B.S., \& Liedahl, D.A. 2000, ApJ, 543, L115

Wu, K., Chanmugam, G., \& Shaviv, G. 1994, ApJ, 426, 664

Wu, K., \& Cropper, M. 2001, MNRAS, 326, 686 